to 2020. We evaluated the change in NIHSS from presentation to hospital discharge as well as mRS at discharge and 3 months. We further evaluated for stent or vessel reocclusion as well as symptomatic hemorrhagic conversion as the cause of clinical decline.

Results There were twenty procedures in nineteen patients during this time period. In three procedures acceptable caliber improvement was achieved using angioplasty alone (15\%), with the other 17 procedures requiring a stent. Fourteen procedures (70\%) resulted in improvement in NIHSS following the procedure and upon discharge. Out of the 6 procedures with worsening clinical outcome, 1 had reocclusion of the lesion, 2 with symptomatic hemorrhagic conversion, and 1 with perforator occlusion. Eleven cases resulted in mRS less than or equal 2 at 3 months and 3 patients had passed away by 3 months.

Conclusion Even though rescue angioplasty with or without stenting can have a high rate of periprocedural morbidity, it leads to marked clinical improvement in the majority of the patients in this selected cohort.

Disclosures K. Khatibi: None. A. Mowla: None. H. Saber: None. L. Ponce Mejia: None. N. Kaneko: None. G. Duckwiler: None. S. Tateshima: None.

\section{E-030 ENDOVASCULAR TECHNIQUES FOR TREATING INTRACRANIAL VERTEBRAL ARTERY DISSECTION - A SINGLE CENTER EXPERIENCE}

H Alhajala*, M Rehman, M Kole, A Chebl, H Marin. Henry Ford Hospital, Detroit, MI

\subsection{6/neurintsurg-2021-SNIS. 126}

Introduction Dissection of the intracranial segment (V4) of the vertebral artery (VAD) is a rare and serious condition. It can present either with ischemic symptoms related to stenosis, thrombosis or embolic phenomenon or with subarachnoid hemorrhage (SAH). Various endovascular techniques have been described for managing VAD. This study was conducted to review our institutional experience with patients with intracranial VAD who required endovascular intervention. Understanding clinical and treatment variables will yield preferred management options and guide our practice.

Methods A retrospective, single-center study to review cases of intracranial vertebral artery dissection treated with endovascular intervention.

Results A total of 20 patients with intracranial vertebral artery dissection were identified. Mean age (SD) was 50.8 (13.1), and $\mathrm{M}: \mathrm{F}$ ratio was 1:1. Presentation was with $\mathrm{SAH}$ in 11 $(55 \%)$ patients, ischemic symptoms in $4(20 \%)$ patients and headache only in $5(25 \%)$ patients. Dissection involved unilateral codominant VA in 10 cases, dominant artery in 2 cases, non-dominant artery in 3 cases and bilateral VAs in 5 cases. Angiographic anatomical review identified 11 VAD (55\%) were distal to the PICA origin, $6(30 \%)$ were at the origin of the PICA, and $3(15 \%)$ were proximal to the PICA origin. Most common dissection etiology was spontaneous in 15 $(75 \%)$ patients followed by fibromuscular dysplasia in $3(15 \%)$ patients and traumatic in $2(10 \%)$ patients. The treatment methods used were parent vessel coil occlusion in $12(60 \%)$ cases, coil embolization of the aneurysm with vessel latency preservation in $5(25 \%)$ cases and flow-diversion in $3(15 \%)$ cases. Periprocedural complications were encountered in 3 cases with symptomatic ischemic symptoms in 2 cases and rebleeding in 1 case (early rerupture after flowdiversion). Median modified Rankin Scale (mRS) on discharge was 2 $(0.5-3.5)$ and on 3 months follow up was 1 (0-2). Two cases of late aneurysmal recanalization were encountered and both cases in patient who were treated with aneurysmal coiling. There were 2 mortalities in the cohort. Both mortalities were due to withdrawal of care: one because of advanced age and the second was due to poor prognosis with massive brainstem infarction.

Conclusion Endovascular treatment provides effective treatment for VAD. In our study, we demonstrated that sacrificing the parent artery with coil occlusion remains an effective and permeant treatment option for ruptured VAD. Risk of complications is low if the occlusion is distal to the origin of PICA as there is adequate collateral blood flow via ipsilateral AICA. Preservation of blood flow to the parent artery with stent assisted coiling or flow diversion stents provides alternative approach for treatment but in the setting of ruptured aneurysms, and the use of antithrombotic carries high rebleeding risk.

Disclosures H. Alhajala: None. M. Rehman: 3; C; Alexion Pharma. M. Kole: None. A. Chebl: None. H. Marin: None.

\section{E-031 VERTEBROBASILAR JUNCTION ANEURYSMS ASSOCIATED WITH SUBCLAVIAN STEAL PHYSIOLOGY: SHOULD AN ASYMPTOMATIC SUBCLAVIAN ARTERY OCCLUSION BE TREATED?}

O Stechishin*, J Corona, T Binyamin, R Novakovic, J Barr, J White, R de Oliveira Sillero, B Welch, L Pride. University of Texas Southwestern Medical Center, Dallas, TX

\subsection{6/neurintsurg-2021-SNIS. 127}

Introduction/Purpose Vertebrobasilar aneurysms are infrequent accounting from $0.5 \%$ of all intracranial aneurysms. Subclavian steal physiology resulting in abnormal flow across the vertebrobasilar junction to supply the distal territory beyond a proximal subclavian artery occlusion or stenosis has been reported as a likely etiology for a subset of vertebrobasilar junction aneurysms. Moreover, subarachnoid hemorrhage due to ruptured vertebrobasilar aneurysms has been reported as a rare initial presentation of otherwise asymptomatic subclavian steal physiology. There are several case reports in the literature of endovascular treatment of vertebrobasilar aneurysms associated with subclavian steal syndrome. However, the long term risk of vertebrobasilar junction aneurysm re-growth and re-rupture in the setting of untreated subclavian steal physiology is unknown. Moreover, few specific recommendations for management of the underlying subclavian artery occlusion in patients with vertebrobasilar aneurysms exist in the literature. Materials and methods We present two cases of patients with asymptomatic subclavian steal physiology initially presenting with ruptured vertebrobasilar junction aneurysms with differing clinical courses.

Results Case 1 is a 70 year old female with a wide-necked 10 $\mathrm{mm}$ vertebrobasilar junction aneurysm successfully treated with stent-assisted coiling. Follow-up imaging demonstrated 1 year stability in complete occlusion of her aneurysm. Her left subclavian origin occlusion remained clinically asymptomatic and was managed conservatively. Case 2 is a 50 year old female presenting initially with a ruptured $4 \mathrm{~mm}$ vertebrobasilar junction aneurysm successfully treated with primary coil 